\title{
Técnicas FT-IR (PAS, UATR e Objetiva ATR) Aplicadas à Caracterização de EPDM Modificada com Plasma
}

\author{
Renata Patrícia dos Santos \\ Indústria Química Una Ltda. \\ Mauro Santos de Oliveira Junior \\ Departamento de Física - Laboratório de Plasmas e Processos - LPP, \\ Instituto Tecnológico de Aeronáutica - ITA \\ Elizabeth da Costa Mattos, Milton Faria Diniz, Rita de Cássia Lazzarini Dutra \\ Divisão de Química - AQI, Instituto de Aeronáutica e Espaço - IAE
}

\begin{abstract}
Resumo: Técnicas FT-IR (UATR, PAS e MIC, com objetiva ATR) foram escolhidas para a caracterização da superfície da borracha de EPDM vulcanizada, após tratamento em plasma de $\mathrm{Ar} / \mathrm{O}_{2}$ e $\mathrm{N}_{2} / \mathrm{H}_{2} /$ Ar gerado em micro-ondas. Após tratamento, grupos oxigenados foram detectados por UATR e MIC/FT-IR, com objetiva ATR para as duas misturas gasosas, e possíveis grupos nitrogenados foram inseridos na superfície das amostras tratadas com plasma de $\mathrm{N}_{2} / \mathrm{H}_{2} / \mathrm{Ar}$. A análise MIC/FT-IR sugeriu a formação de grupos em uma camada mais externa, para as amostras tratadas com plasma de $\mathrm{N}_{2} / \mathrm{H}_{2} / \mathrm{Ar}$, sendo possível observar a redução na intensidade das bandas da absorção do $\mathrm{CH}_{2}$ e $\mathrm{CH}_{3}$, o que pode estar relacionado ao fato de o nitrogênio ser um gás menos permeável que o oxigênio. Diferentes velocidades, $0,05 \mathrm{~cm} \cdot \mathrm{s}^{-1} \mathrm{e} 0,2 \mathrm{~cm} \cdot \mathrm{s}^{-1}$, foram utilizadas na análise PAS para avaliar a superfície após tratamento, e apenas para a velocidade $0,05 \mathrm{~cm} \cdot \mathrm{s}^{-1} \mathrm{com}$ plasma de $\mathrm{Ar} / \mathrm{O}_{2}$ alterações espectroscópicas foram detectadas. A redução na medida de ângulo de contato e o aumento na resistência ao descascamento da colagem indicaram melhoras nas propriedades adesivas da superfície. Falhas de adesão foram observadas entre a interface do filme de adesivo de PU e da borracha de EPDM, e confirmados por UATR/FT-IR.
\end{abstract}

Palavras-chave: EPDM, plasma, caracterização de superfície, UATR/FT-IR, PAS, MIC.

\section{FT-IR Techniques (PAS, UATR and Objective ATR) Applied to the Characterization of Plasma-Modified Surface of EPDM}

Abstract: The FT-IR techniques UATR, PAS and MIC with ATR objective were chosen to characterize the surface of EPDM rubber vulcanized after treatment in $\mathrm{Ar} / \mathrm{O}_{2}$ or $\mathrm{N}_{2} / \mathrm{H}_{2} / \mathrm{Ar}$ plasma generated in a microwave oven. After treatment, oxygenated groups were detected by UATR and MIC-FT-IR, with ATR objective for the two gas mixtures, and possible nitrogenous groups were inserted on the surface after the plasma treatment with $\mathrm{N}_{2} / \mathrm{H}_{2} /$ Ar. The MIC/FT-IR analysis suggested that groups were formed on an outermost layer, inferred from a reduced intensity of the $\mathrm{CH}_{2}$ and $\mathrm{CH}_{3}$ absorption bands for the treatment in $\mathrm{N}_{2} / \mathrm{H}_{2} / \mathrm{Ar}$ plasma, probably because nitrogen is less permeable than oxygen. Different speeds with PAS analyses $\left(0.05 \mathrm{~cm} . \mathrm{s}^{-1}\right.$ and $\left.0.2 \mathrm{~cm} . \mathrm{s}^{-1}\right)$ were used to evaluate the surface after treatment. Only for the speed $0.05 \mathrm{~cm} \cdot \mathrm{s}^{-1}$ in $\mathrm{Ar} / \mathrm{O}_{2}$ plasma were spectroscopic changes detected. The decrease in the contact angle and the increased resistance to peel indicated improvements in the bonding adhesive properties of the surface. Bonding failure was observed between the adhesive PU film and the EPDM rubber, which was confirmed by UATR/FT-IR.

Keywords: EPDM, plasma, surface characterization, UATR/FT-IR, PAS, MIC.

\section{Introdução}

O EPDM (etileno-propileno-dieno) é uma das borrachas sintéticas que tem apresentado rápido crescimento no mercado, podendo ser, amplamente, utilizada em vários setores ${ }^{[1]}$, como modificador termoplástico, isolante elétrico, isolante acústico, entre outros $^{[2]}$, devido à sua cadeia hidrocarbônica saturada e à presença de duplas ligações na cadeia lateral ${ }^{[1]}$. A borracha de EPDM possui propriedade de volume superior em relação à propriedade de superfície, o que é comum entre os materiais poliméricos, que em geral, não conferem excelentes propriedades para ambos, em aplicações especifica. O processo de vulcanização, geralmente, piora as propriedades de superfície, pois durante a formação das ligações cruzadas, ingredientes como ácido esteárico, enxofre, entre outros, migram para a superfície e criam uma barreira entre o adsorvente e o adsorvido. Desta forma, é necessária a alteração da superfície para que se consiga uma boa adesão ${ }^{[3,4]}$.

Entre as diversas técnicas citadas em literatura, o plasma tem sido relatado como um processo para a modificação da superfície da borracha de $\mathrm{EPDM}^{[3,4]}$. O plasma é constituído, quimicamente, de espécies ativas, representadas por uma larga variedade de componentes, como partículas excitadas e ionizadas, ambas atômicas e

Autor para correspondência: Rita de Cássia Lazzarini Dutra, Divisão de Química - AQI, Instituto de Aeronáutica e Espaço - IAE, Av. Marechal do Ar Eduardo Gomes, 50, Vila das Acácias, CEP 12228-904, São José dos Campos, SP, Brasil, e-mail: ritarcld@iae.cta.br 
moleculares, fótons, radicais. $\mathrm{O}$ ambiente reativo, quando em contato com a superfície do polímero, leva a reações físico-químicas, que quebram as ligações, gerando sítios ativos, grupos polares e produtos resultantes da quebra da cadeia na superfície ${ }^{[5]}$

Dentre as técnicas de caracterização FT-IR, encontradas na literatura, ATR (reflexão total atenuado) é a mais utilizada, para identificar os grupos funcionais formados na superfície dos polímeros tratados com plasma, tanto para borracha como para plásticos ${ }^{[3,6-9]}$. Entretanto, outras técnicas espectroscópicas de reflexão podem ser usadas, tais como UATR (universal reflexão total atenuada) e microscopia com objetiva ATR.

Santos e colaboradores, em trabalho recente, utilizaram o acessório UATR (universal reflexão total atenuada) para caracterizar a superfície da borracha de EPDM tratada com plasma de $\mathrm{Ar}, \mathrm{Ar} / \mathrm{O}_{2}, \mathrm{~N}_{2} / \mathrm{O}_{2}$ e $\mathrm{N}_{2} /$ $\mathrm{H}_{2}$, gerado por micro-ondas, onde grupos oxigenados e especificamente, para os tratamentos contendo nitrogênio $\left(\mathrm{N}_{2} / \mathrm{O}_{2}\right.$ e $\left.\mathrm{N}_{2} / \mathrm{H}_{2}\right)$, possíveis grupos nitrogenados foram detectados ${ }^{[10]}$. Em complemento ao estudo e levando em consideração a dificuldade na disponibilidade do equipamento XPS, e também por ser uma técnica que exige longo tempo de análise, motivou a busca do estudo das diferentes técnicas disponíveis FT-IR, como MIC com objetiva e PAS, além do UATR, visando a possibilidade de avaliação dos diferentes graus de amostragem das mesmas, pois a variação do tipo do gás utilizado nos tratamentos por plasma apresenta graus de permeabilidade diferentes.

A objetiva ATR é citada na literatura para a caracterização de diferentes formulações de borrachas com negro de fumo e para a determinação da oxidação das mesmas envelhecidas. Tal acessório de microscopia FT- IR (MIC) permitiu um contato pontual da amostra em pequenas áreas ${ }^{[11]}$.

A espectroscopia fotoacústica (PAS), outra técnica FT-IR, permite variar as diferentes velocidades de modulação da frequência e assim obter espectros de diferentes camadas da amostra. Velocidades mais lentas, neste caso, atingem uma camada mais interna da superfície. Já o uso de velocidades maiores, permite a analise de uma camada mais superficial ${ }^{[12]}$.

Para este trabalho, as mais diferenciadas técnicas FT-IR: PAS, MIC com objetiva ATR e UATR foram utilizadas, levando-se em conta os seus diferentes graus de profundidade, na análise da borracha de EPDM, tratada com duas diferentes misturas gasosas $\left(\mathrm{Ar} / \mathrm{O}_{2}\right.$ e N $\mathrm{N}_{2} /$ $\mathrm{H}_{2} / \mathrm{Ar}$ ), visando identificar os possíveis grupos funcionais formados na superfície. As melhorias nas propriedades de superfície foram avaliadas por meio do ensaio de resistência ao descascamento da colagem do sistema EPDM x PU, e também por goniometria.

\section{Materiais}

Foi usada a borracha de EPDM com dieno (etilenonorboneno), com baixo teor de dieno $(<1 \%)$ e $42 \%$ de etileno, fornecida pela Dow Chemical Company. O processamento foi realizado pela Flexlab Consultoria e Treinamento Ltda. A formulação contém 100 phr (parte por cem de borracha) da borracha de EPDM,
$1 \mathrm{phr}$ de negro de fumo, $2 \mathrm{phr}$ de óleo parafínico, $1 \mathrm{phr}$ de monosulfeto de tetrametiltiuram (TMTM), 0,5 phr de mercaptonobenzotiazol (MBT), 0,5 phr de ácido esteárico, $2 \mathrm{phr}$ de óxido de zinco e 1 phr de enxofre. A borracha foi vulcanizada a temperatura de $190{ }^{\circ} \mathrm{C}$ por 5 minutos.

\section{Limpeza das Amostras em Ultrassom}

A borracha de EPDM foi lavada usando o ultrassom em uma frequência de $37 \mathrm{kHz}$ em tanque de aço, condições estas usuais de limpeza ${ }^{[13]}$. Os corpos de prova foram imersos em uma solução de Unalimp WB 2151 (detergente) fornecida pela Indústria Química Una Ltda. A solução diluída (4:21 - detergente : água) foi levada ao tanque ultrassônico por $300 \mathrm{~s}$ a $60{ }^{\circ} \mathrm{C}$. Após limpeza, as amostras foram imersas em água, também por $300 \mathrm{~s}$ a $60{ }^{\circ} \mathrm{C}$, e na sequencia, foram colocados em uma estufa (Tecnal, modelo TE-394/2) a $64{ }^{\circ} \mathrm{C}$ por 20 minutos. O tratamento por plasma foi realizado, após, aproximadamente, 18 horas de limpeza.

\section{Medidas de Ângulo de Contato}

As medidas de ângulo de contato foram realizadas no equipamento Standard Digital Automed Goniometer modelo 500-00 da Ramé-Hart Inc. O cálculo foi obtido pelo software DROPimage Advanced. Foram obtidas de 4 a 7 medidas de ângulo de contato, em diferentes pontos, para a água deionizada.

\section{Tratamento com Plasma}

O plasma para este estudo foi gerado em um reator de processos a plasma de micro-ondas, que consiste em uma câmara de secção retangular de $230 \mathrm{~mm}$ de largura, $80 \mathrm{~mm}$ de altura e $500 \mathrm{~mm}$ de profundidade, com porta para observação, inserção e retirada das amostras. Há uma bomba de vácuo mecânico para redução da pressão interna da câmara (Edwards modelo E2M18). A energia necessária para o funcionamento do equipamento é fornecida pela rede elétrica, a qual é amplificada para o magnetron gerando ondas na frequência de $2,45 \mathrm{GHz}$. Os tratamentos foram realizados usando, misturas de $\mathrm{Ar} / \mathrm{O}_{2}$ (1:3) e $\mathrm{N}_{2} / \mathrm{H}_{2} / \mathrm{Ar}$ (1:4:1) sob o fluxo total de $24 \mathrm{sccm}$ (centímetros cúbicos por minuto) e pressão de $250 \mathrm{mTorr}$, e $50 \mathrm{sccm}$ e pressão 300 mTorr, respectivamente. Para os dois sistemas, o tempo de tratamento foi de $120 \mathrm{~s}$.

\section{Avaliação da Adesão do EPDM/Adesivo de PU/PVC}

Este ensaio tem por objetivo avaliar as propriedades adesivas da borracha de EPDM, tratada com plasma, por intermédio dos valores de resistência ao descascamento do sistema EPDM/adesivo de PU/PVC. O adesivo foi aplicado na borracha de EPDM e no PVC. O PVC foi escolhido para formar a junta adesiva, pois já é conhecida sua boa interface com adesivo de PU. A superfície do PVC foi limpa com acetona. Os testes de resistência as descascamento da colagem foram avaliados no dinamômetro Emic DL 500, com célula de carga de 50 kgf e velocidade de afastamento das garras de $100 \mathrm{~mm} /$ min. As dimensões dos corpos de prova para realizar as colagens foram de $20 \mathrm{~mm}$ de largura e $50 \mathrm{~mm}$ de 
comprimento. O número de coros de prova utilizado para cada tratamento foi de cinco amostras.

\section{Análise por FT-IR}

As análises foram realizadas noIAE/AQI, utilizando-se o espectrômetro FT-IR Spectrum One PerkinElmer (resolução $4 \mathrm{~cm}^{-1}$, ganho $1,4000-550 \mathrm{~cm}^{-1}$ ), com o acessório universal UATR, contendo ZnSe e diamante e torque de $100 \mathrm{~N}$.

Para as análises por MIC, com objetiva ATR, e PAS, foi utilizado FT-IR Spectrum 2000 PerkinElmer. Com a objetiva ATR, as condições usadas foram: faixa espectral de 4000 a $700 \mathrm{~cm}^{-1}$, resolução 8 e 128 varreduras. $\mathrm{Na}$ análise PAS, as condições foram: resolução 4 , velocidades 0,05 e $0,2 \mathrm{~cm} \cdot \mathrm{s}^{-1}, 4000$ a $400 \mathrm{~cm}^{-1}, 32$ a 128 varreduras e ganho 6 no amplificador.

\section{Resultados e Discussão}

\section{Análises UATR/FT-IR da superfície da borracha de EPDM, após tratamento com plasma de $\mathrm{Ar} / \mathrm{O}_{2}$ e $\mathrm{N}_{2} / \mathrm{H}_{2} / \mathrm{Ar}$}

A Figura 1 apresenta os espectros UATR/FT-IR da borracha não tratada (Figura 1a) e da borracha tratada com plasma de $\mathrm{Ar} / \mathrm{O}_{2}$ e $\mathrm{N}_{2} / \mathrm{H}_{2} / \mathrm{Ar}$, e em ambos os tratamentos, grupos oxigenados foram formados. $\mathrm{Na}$ Figura 1b, observa-se a presença do $\mathrm{OH}$ na faixa de 3500 a $3200 \mathrm{~cm}^{-1}$, C-O entre $1200-1000 \mathrm{~cm}^{-1}$ e $\mathrm{OH}$ entre 770 a $650 \mathrm{~cm}^{-1[14]}$. Na Figura 1c, há formação de grupos oxigenados e possíveis grupos nitrogenados, pois há possibilidade de sobreposição destas bandas, devido suas absorções ocorrerem. Desta forma, possíveis grupos

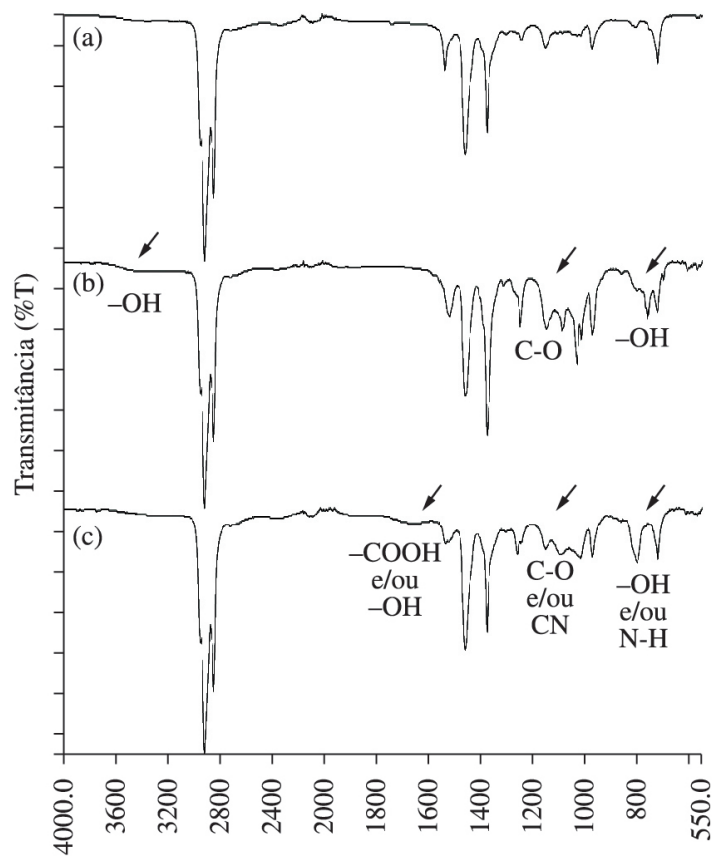

Números de onda $\left(\mathrm{cm}^{-1}\right)$

Figura 1. Espectros UATR/FT-IR das amostras da borracha de EPDM: (a) não tratada, tratada com plasma de (b) $\mathrm{Ar} / \mathrm{O}_{2}$ e (c) $\mathrm{N}_{2} / \mathrm{H}_{2} /$ Ar. foram formados como: $\mathrm{COOH}$ e/ou $\mathrm{OH}$ entre 1700 a $1600 \mathrm{~cm}^{-1}$, C-O e/ou C-N entre 1200 a $1000 \mathrm{~cm}^{-1[15]} \mathrm{e} \mathrm{OH}$ e/ou N-H entre 770 a $550 \mathrm{~cm}^{-1[14]}$. Entretanto, é relatado na literatura ${ }^{[16]}$ que o oxigênio, sendo um gás que tem alta permeabilidade, possibilita sua migração para uma camada abaixo da superfície, permitindo a detecção de grupos oxigenados por FT-IR.

Análises MIC/FT-IR, com objetiva ATR, da superfície da borracha de EPDM, após tratamento com plasma de $\mathrm{Ar} / \mathrm{O}_{2}$ e $\mathrm{N}_{2} / \mathrm{H}_{2} / \mathrm{Ar}$

Nos espectros MIC/FT-IR, utilizando a objetiva ATR, da borracha tratada com plasma de $\mathrm{Ar} / \mathrm{O}_{2}(1: 3)$, foram observadas alterações espectroscópicas, como demonstrado na Figura 2b, na região de 1700 e $1600 \mathrm{~cm}^{-1}$, provavelmente atribuída à presença de grupo $\mathrm{C}=\mathrm{O}$.

Alterações espectroscópicas para a borracha de EPDM tratada com $\mathrm{N}_{2} / \mathrm{H}_{2} / \mathrm{Ar}$ (1:4:1), foram observadas entre $1800 \mathrm{e} 1700 \mathrm{~cm}^{-1}$ e entre 1650 e $1550 \mathrm{~cm}^{-1}$ relacionadas, respectivamente, às absorções $\mathrm{COOH} / \mathrm{C}=\mathrm{O}$ e $\mathrm{OH}$ e/ou NH. Banda de $\mathrm{OH}$ e/ou NH pode, também ser observada na região de 850 e $750 \mathrm{~cm}^{-1}$ (Figura 2c). A intensidade das bandas $\mathrm{CH}_{2}$, em $2911 \mathrm{~cm}^{-1}$ e $2849 \mathrm{~cm}^{-1}$ diminuíram, conforme Figura 2c. Em acordo com Moraes e colaboradores, redução na intensidade das bandas $\mathrm{CH}_{2}$ e $\mathrm{CH}_{3}$ também foram observadas neste estudo, para os tratamentos em plasma de $\mathrm{N}_{2} / \mathrm{Ar}$ da borracha de EPDM, devido à formação de grupos funcionais contendo nitrogênio, este comportamento foi também, observado em alguns casos, neste estudo, onde o nitrogênio foi usado para o tratamento ${ }^{[6]}$.

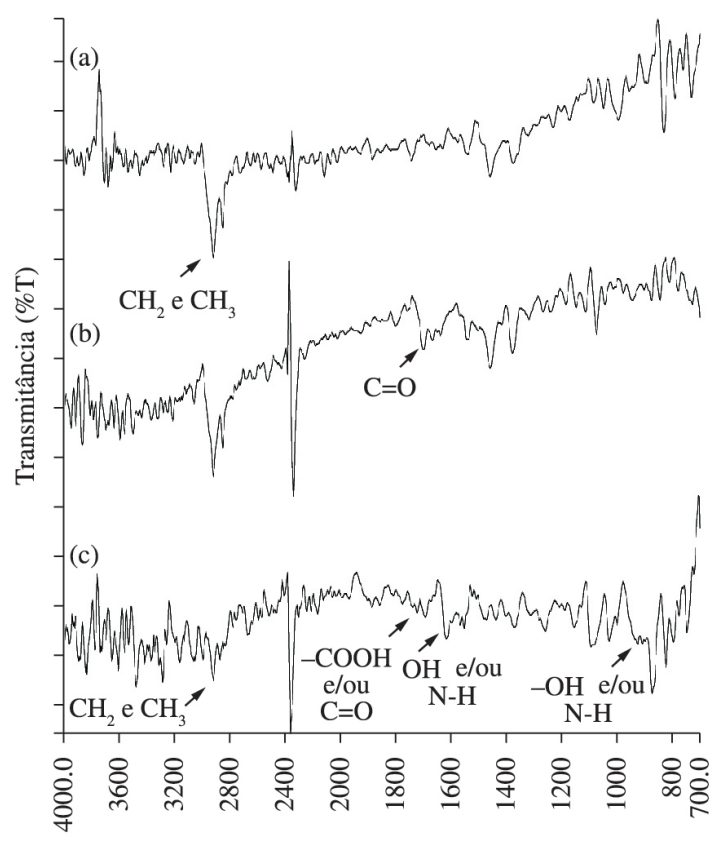

Números de onda $\left(\mathrm{cm}^{-1}\right)$

Figura 2. Espectros MIC/FT-IR, com objetiva ATR, das amostras da borracha de EPDM: (a) não tratada, tratada por plasma de (b) $\mathrm{Ar} / \mathrm{O}_{2}$ e (c) $\mathrm{N}_{2} / \mathrm{H}_{2} / \mathrm{Ar}$. 
Análises PAS/FT-IR da superfície da borracha de EPDM, após tratamento com plasma de $\mathrm{Ar} / \mathrm{O}_{2}$ e $\mathrm{N}_{2} / \mathrm{H}_{2} / \mathrm{Ar}$

Espectros da borracha de EPDM tratada em $\mathrm{Ar} / \mathrm{O}_{2}$ e $\mathrm{N}_{2} / \mathrm{H}_{2} / \mathrm{Ar}$ foram obtidos por PAS, com velocidades de $0,05 \mathrm{~cm} \cdot \mathrm{s}^{-1}$ e $0,2 \mathrm{~cm} \cdot \mathrm{s}^{-1}$ (Figuras 3 e 4 ). Alterações espectroscópicas, neste caso, foram possíveis de serem detectadas para o tratamento em $\mathrm{Ar} / \mathrm{O}_{2}$ para a velocidade de $0,05 \mathrm{~cm} \cdot \mathrm{s}^{-1}$. Grupos funcionais oxigenados foram provavelmente inseridos na superfície, pois mudanças espectroscópicas foram encontradas em $3753 \mathrm{~cm}^{-1}$ (OH livre), $1153 \mathrm{~cm}^{-1}(\mathrm{CO})$ e $968 \mathrm{~cm}^{-1}(\mathrm{OH})$ (Figura 3b). Não foram observadas alterações espectroscópicas para tratamento em $\mathrm{Ar} / \mathrm{O}_{2}$, velocidade de $0,2 \mathrm{~cm} \cdot \mathrm{s}^{-1}$ (Figura $3 \mathrm{~b}$ ) para o tratamento em $\mathrm{N}_{2} / \mathrm{H}_{2} / \mathrm{Ar}$, nas duas velocidades, $0,05 \mathrm{~cm} \cdot \mathrm{s}^{-1}$ e $0,2 \mathrm{~cm} \cdot \mathrm{s}^{-1}$ (Figuras $4 \mathrm{~b}$ e $4 \mathrm{~d}$ ). Uma provável explicação pode estar no fato de que, conforme a literatura, e já citado anteriormente, grupos oxigenados e nitrogenados atingem camadas diferentes, sendo esperada uma modificação na superfície mais interna, para tratamentos com plasma contendo oxigênio, em acordo com o observado na análise PAS, em velocidades menores.

\section{Medidas de ângulo de contato}

As medidas foram realizadas gotejando água sobre a superfície da borracha e o ângulo de contato resultante, fio o formado entre a superfície do polímero e a curva da gota de água ${ }^{[17]}$. Foi observada uma redução significativa

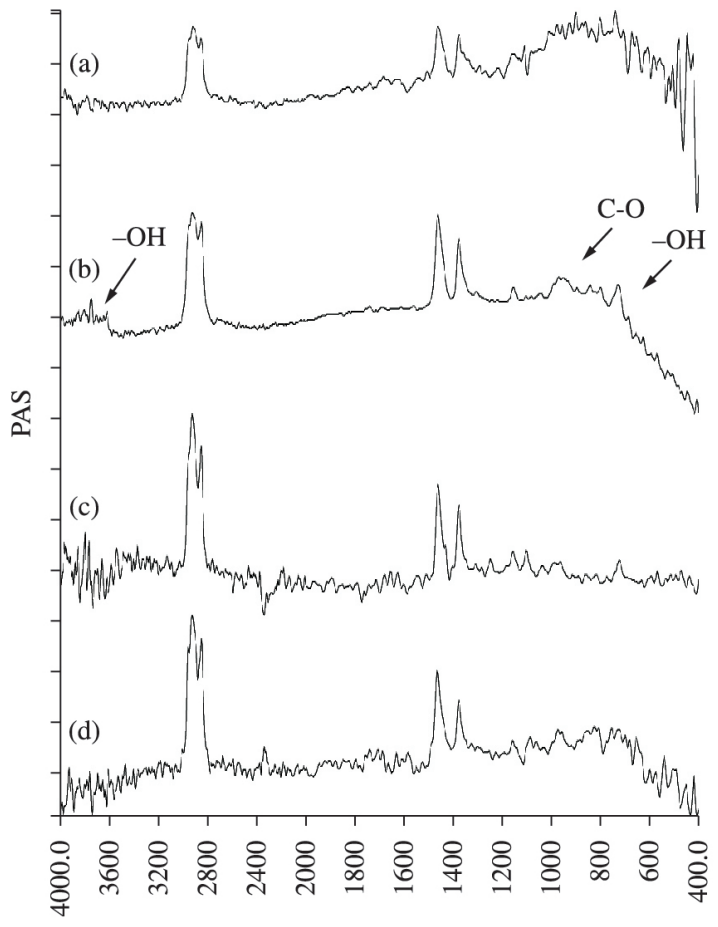

Números de onda $\left(\mathrm{cm}^{-1}\right)$

Figura 3. Análises por PAS/FT-IR da borracha de EPDM não tratada com (a) velocidades de $0,05 \mathrm{~cm} \cdot \mathrm{s}^{-1} \mathrm{e}$ (c) de $0,2 \mathrm{~cm} \cdot \mathrm{s}^{-1}$, e da borracha tratada com plasma de $\mathrm{Ar} / \mathrm{O}_{2}$ (b) velocidades de 0,05 e (d) $0,2 \mathrm{~cm} \cdot \mathrm{s}^{-1}$. nas medidas do ângulo de contato, após tratamento com plasma de $\mathrm{Ar} / \mathrm{O}_{2}$ e $\mathrm{N}_{2} / \mathrm{H}_{2} / \mathrm{Ar}$ (Tabela 1) de $72.31 \%$ e $67.24 \%$ respectivamente. Esta redução pode ser atribuída à inserção de grupos polares na superfície da amostra, detectados por FT-IR.

A redução no ângulo de contato resulta em um consequente aumento no trabalho de adesão $\left(\mathrm{W}_{\mathrm{A}}\right)$ foi calculado a partir da equação de Young-Dupré (Equação 1), relacionando a tensão superficial do líquido $\left(\gamma^{\mathrm{LV}}\right)$ e o ângulo de contato do líquido formado sobre a superfície ${ }^{[18,19]}$. O trabalho de adesão atingirá seu valor máximo quando houver completa molhabilidade, neste caso, da água sobre a superfície, pois o trabalho de adesão líquido-sólido iguala, ou supera o trabalho de coesão do líquido $^{[20]}$.

$$
\mathrm{W}_{\mathrm{A}}=\gamma^{\mathrm{LV}} \cdot[\cos (\theta)+1]
$$

\section{Medidas de resistência ao descascamento da colagem/ UATR-FT-IR}

Por meio do ensaio de resistência ao descascamento da colagem utilizado para avaliar a interface do adesivo

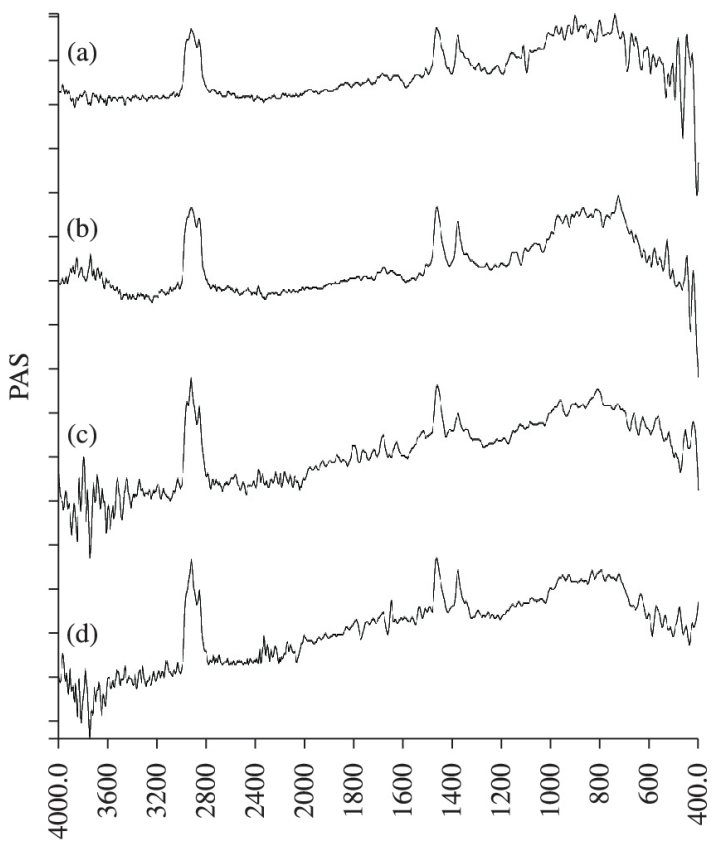

Números de onda $\left(\mathrm{cm}^{-1}\right)$

Figura 4. Análises por PAS/FT-IR da borracha de EPDM não tratada com (a) velocidades de $0,05 \mathrm{~cm} \cdot \mathrm{s}^{-1} \mathrm{e}$ (c) de $0,2 \mathrm{~cm} \cdot \mathrm{s}^{-1}$, e da borracha tratada com plasma de $\mathrm{N}_{2} / \mathrm{H}_{2} / \mathrm{Ar}$ (b) velocidades de 0,05 e (d) $0,2 \mathrm{~cm} \cdot \mathrm{s}^{-1}$.

Tabela 1. Resultados das medidas de ângulo de contato e trabalho de adesão após tratamento com plasma.

\begin{tabular}{ccc}
\hline $\begin{array}{c}\text { Condições do } \\
\text { tratamento } \\
\text { Gás/tempo/fluxo }\end{array}$ & $\begin{array}{c}\text { Ângulo de contato } \\
\left({ }^{\circ}\right)\end{array}$ & $\begin{array}{c}\text { Trabalho de adesão } \\
(\mathbf{m N} / \mathbf{m})\end{array}$ \\
\hline $\mathrm{Não}$ tratada & $122,42 \pm 0,52$ & $33,80 \pm 0,80$ \\
$\mathrm{Ar} / \mathrm{O}_{2}$ & $33,89 \pm 2,33$ & $133,20 \pm 1,70$ \\
$\mathrm{~N}_{2} / \mathrm{H}_{2} / \mathrm{Ar}$ & $40,10 \pm 2,30$ & $128,50 \pm 1,90$ \\
\hline
\end{tabular}


de poliuretano versus a borracha de EPDM, apresentado na Figura 5, observa-se que há melhoras nas propriedades adesivas após tratamento com plasma de $\mathrm{Ar} / \mathrm{O}_{2}$ e $\mathrm{N}_{2} / \mathrm{H}_{2} / \mathrm{Ar}$. Esta melhora pode ser atribuída aos grupos funcionais polares, oxigenados e/ou possíveis grupos nitrogenados, neste ultimo caso, apenas quando tratado com $\mathrm{N}_{2} / \mathrm{H}_{2} / \mathrm{Ar}$, foram formados na superfície da borracha.

$\mathrm{O}$ adesivo de poliuretano utilizado para formar a junta adesiva, possui em sua composição um aditivo, branqueador óptico, que permite a visualização do seu filme quando exposto sob a luz negra. Conforme demonstrado na Figura 6, têm-se os corpos de prova da borracha de EPDM sem adesivo (Figura 6a), com o adesivo (Figura 6b), e neste último, visualiza-se o filme do adesivo de PU azulado. A superfície do corpo de prova (borracha), após ensaio de resistência ao descascamento da colagem (Figura 6c) é similar ao sem adesivo e assim é possível detectar a falha na junta adesiva. Embora melhoras nas propriedades adesivas tenham sido observadas, há falhas de adesão entre a interface do filme do adesivo de PU e da borracha de EPDM.

Para melhor averiguar a junta adesiva borracha de EPDM tratada/adesivo de PU/PVC, espectros UATR/FT-IR foram obtidos dos corpos de prova após o ensaio de resistência ao descascamento da colagem. Na

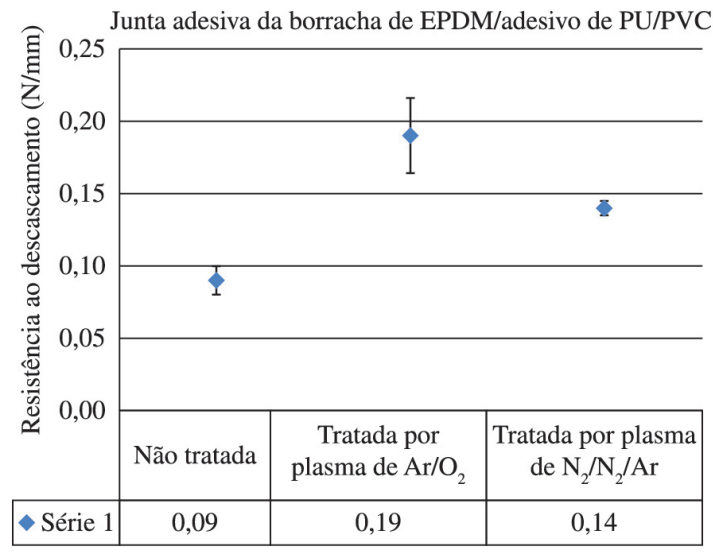

Figura 5. Ensaio de resistência ao descascamento da colagem da junta EPDM/Adesivo de PU/PVC, com a borracha não tratada e tratada com plasma de $\mathrm{Ar} / \mathrm{O}_{2}$ e $\mathrm{N}_{2} / \mathrm{H}_{2} / \mathrm{Ar}$.

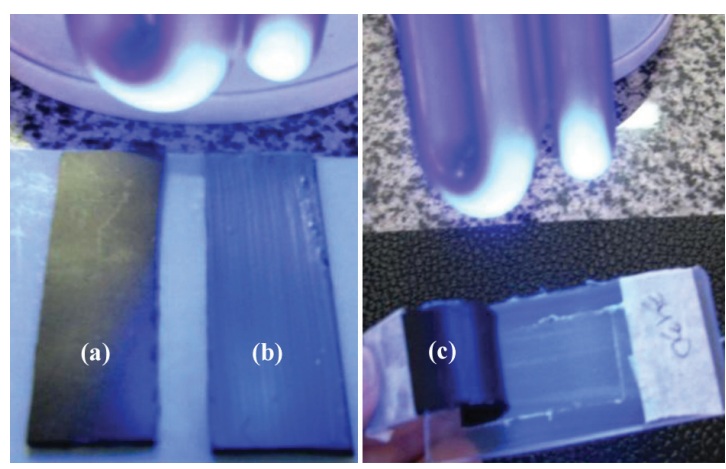

Figura 6. Corpos de prova exposto sob a luz negra da (a) borracha sem adesivo, (b) da borracha com adesivo de PU e (c) após o ensaio de resistência ao descascamento da colagem.
Figura 7, têm-se os espectros da superfície da borracha de EPDM tratada com plasma de $\mathrm{Ar} / \mathrm{O}_{2}$ (Figura 7a) e $\mathrm{N}_{2} / \mathrm{H}_{2} / \mathrm{Ar}$ (Figura 7b) mais o filme do adesivo de PU, e apenas do filme de PU (Figura 7c), e absorções que caracterizam o PU foram detectadas: $\mathrm{NH}$ em $3388 \mathrm{~cm}^{-1}$ $(\mathrm{NH}), 3000-2840 \mathrm{~cm}^{-1}(\mathrm{C}-\mathrm{H}), 1800-1700 \mathrm{~cm}^{-1}(\mathrm{C}=\mathrm{O})$, $1520 \mathrm{~cm}^{-1}(\mathrm{C}-\mathrm{N}+\mathrm{N}-\mathrm{H}), 1262 \mathrm{~cm}^{-1}$ (N-C ou C-O-C),

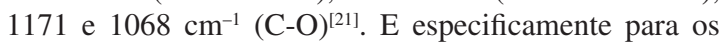
corpos de prova da borracha de EPDM tratados, tanto com $\mathrm{Ar} / \mathrm{O}_{2}$ como para $\mathrm{N}_{2} / \mathrm{H}_{2} / \mathrm{Ar}$, os espectros apresentaram um alargamento observado na região entre $1800-1700 \mathrm{~cm}^{-1}$ referente ao grupo $\mathrm{C}=\mathrm{O}$, e $\mathrm{OH}$ e/ou $\mathrm{N}-\mathrm{H}(\mathrm{N}-\mathrm{H}$ apenas para o tratamento com a mistura gasosa que contem nitrogênio) entre 770 a $550 \mathrm{~cm}^{-1}$, que possivelmente teve contribuição pela funcionalização da superfície com o tratamento com plasma.

Nos espectros obtidos após o ensaio de resistência ao descascamento da colagem, apenas absorções da borracha de EPDM foram detectados, exceto pela presença da banda de $\mathrm{OH}$ na região entre 770 a $550 \mathrm{~cm}^{-1[15]}$ para as duas misturas gasosas utilizadas, e/ou possivelmente $\mathrm{N}-\mathrm{H}$ na mesma região para o tratamento com $\mathrm{N}_{2} / \mathrm{H}_{2} / \mathrm{Ar}$, grupos estes ainda referentes ao tratamento com plasma. Desta forma, a falha de adesão entre a interface do EPDM x PU é comprovada.

De forma similar, Sánchez e colaboradores detectaram por meio da técnica FT-IR (ATR) falhas

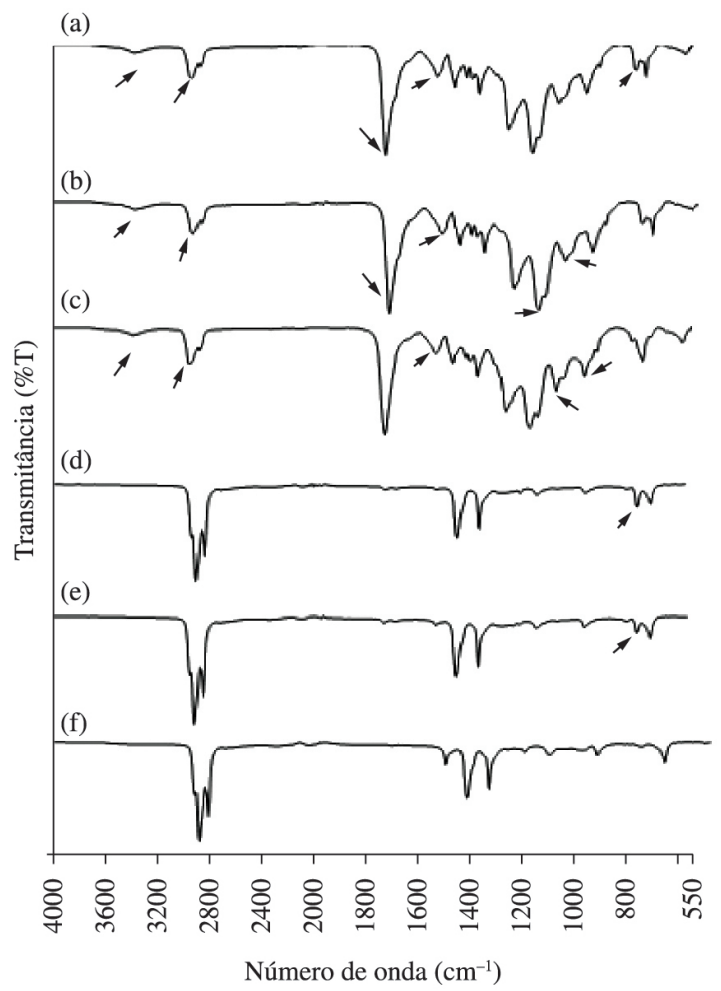

Figura 7. Espectros da borracha de EPDM tratada por plasma de (a) $\mathrm{Ar} / \mathrm{O}_{2}$ com uma camada de adesivo de $\mathrm{PU}$ e (b) $\mathrm{N}_{2} / \mathrm{H}_{2} / \mathrm{Ar}$ com uma camada de adesivo de PU, espectros do (c) adesivo de PU e borracha de EPDM tratada por plasma de (d) $\mathrm{Ar} / \mathrm{O}_{2} \mathrm{e}$ (e) $\mathrm{N}_{2} / \mathrm{H}_{2} /$ Ar após ensaio de resistência ao descascamento. E o espectro da borracha de EPDM (f) não tratada. 
de adesão na interface da borracha de SBR (estirenobutadieno) tratada com corona/adesivo de PU/couro. O espectro da superfície da borracha de SBR foi obtido após o ensaio de resistência ao descascamento da colagem, que evidenciou apenas absorções da SBR. Já o espectro obtido da borracha de SBR com adesivo de PU, apresentou absorções referentes ao adesivo de PU. Comparando os espectros, comprova-se a falha na junta adesiva ${ }^{[21]}$.

\section{Conclusão}

Grupos oxigenados foram detectados utilizando o acessório UATR para os tratamentos com plasma de $\mathrm{Ar} / \mathrm{O}_{2}$ e $\mathrm{N}_{2} / \mathrm{H}_{2} /$ Ar e possível grupo nitrogenado especificamente para o tratamento em plasma de $\mathrm{N}_{2} / \mathrm{H}_{2} /$ Ar. Grupos oxigenados também foram observados por meio da técnica MIC/FT-IR com objetiva ATR, porém apresentou uma contribuição adicional, onde foi observada uma redução nas bandas do grupo $\mathrm{CH}_{2}$ e $\mathrm{CH}_{3}$ para o tratamento com plasma da mistura gasosa $\mathrm{N}_{2} / \mathrm{H}_{2} / \mathrm{Ar}$, o que sugere que o MIC/FT-IR detecta uma camada mais superficial, já que o nitrogênio é um gás menos permeável. Já a análise PAS, mostrou alterações espectroscópicas apenas para o tratamento com $\mathrm{Ar} / \mathrm{O}_{2}$ com a velocidade mais lenta $\left(0,05 \mathrm{~cm} \cdot \mathrm{s}^{-1}\right)$, que detecta uma camada mais interna, sendo observados grupos oxigenados. As reduções no ângulo de contato encontradas nos tratamentos para as duas misturas gasosas estão relacionadas aos grupos polares inseridos na superfície. Esta melhora nas propriedades adesivas também foi confirmada pelo aumento nos resultados obtidos no ensaio de resistência ao descascamento da colagem.

Embora observou-se melhoras nas propriedades adesivas da superfície da borracha de EPDM tratada com plasma de $\mathrm{Ar} / \mathrm{O}_{2}$ e $\mathrm{N}_{2} / \mathrm{H}_{2} / \mathrm{Ar}$, falhas na junta adesiva foram detectas por FT-IR. Os espectros obtidos após o ensaio de resistência ao descascamento da colagem apresentaram apenas absorções referentes à borracha de EPDM, com exceção apenas da presença do grupo $\mathrm{OH}$ na superfície dos corpos de prova da borracha tratados via plasma e não está relacionado à presença do filme do adesivo de PU.

\section{Referências Bibliográficas}

1. Zhao, Q.; Li, X.; Gao, J. - Poly. Degrad. Stab.,93, p.692(2010). http://dx.doi.org/10.1016/j.polymdegradstab.2007.12.009

2. Babbit, R. O. - "The Vanderbilt Rubber Handbook", R. T. Company, Inc. Vanderbilt Norwalk, CT (1978). PMid:729323.

3. Basak, G. C.; Banddyopadhyay, A.; Neogi, S.; Bhowmick, A. K. - Appl. Surf. Sci., APSUSC-20806, p.1 (2010).
4. Basak, G. C.; Banddyopadhyay, A.; Bhradwaj, Y. K.; Sabharwal, S.; Bhowmick, A. K. - J. Adhesion, 86, p.314 (2010). http://dx.doi.org/10.1080/00218460903479305

5. Borcia, C.; Borcia, G. \& Dumitrascu, N. - Journ. Phys., 56, p.224 (2011).

6. Moraes, J. H.; Maciel, H. S.; Sobrinho, A. S. S. \& Massi, M. - J. Optoelectron. Adv. M., 9, p.475 (2007).

7. Weon, J-I. \& Choi, K. Y. - Macromol. Res., 17, p.891 (2009).

8. Costa, T. H. F.; Feitor, M. C.; Alves Junior, C. \& Bezerra, C. M. - Rev. Matéria, 13, p.66 (2008).

9. Morent, R.; Geyter, N.; Leys, C.; Gengembre, L. \& Payen, E. - Surf. Interface Anal., 40, p.597 (2008). http://dx.doi. org/10.1002/sia.2619

10. Santos, R. P.; Oliveira Junior, M. S.; Mattos, E. C.; Diniz, M. F. \& Dutra, R. C. L. - Polímeros, 22, p.1 (2012).

11. Do, T. T.; Celina, M. \& Fredericks, P. M. - Polym. Degrad. Stab., 77, p.418 (2002).

12. Nogueira, L. M.; Dutra, R.C.L.; Diniz, M. F.; Pires, M.; Evangelista, M.; Santana, F. A.; Tomasi, L.; Santos, P. \& Nonemacher, R. - Polímeros, 17, p.61 (2007).

13. Peters, D. - J Mater Chem., 6, p.1616 (1996).

14. Smith, A. L. - "Applied Infrared Spectroscopy”, John Wiley \& Sons, New York(1979).

15. Silverstein, R. M. \& Webster, F. X. -"Identificação Espectrométrica de Compostos Orgânicos”, p.84, 6. ed., LTC - Livros Técnicos e Científicos Editora S. A., Rio de Janeiro (2000).

16. Viadaurre, E. F. C.; Achete, C. A.; Gallo, F.; Garcia, D.; Simão, R. \& Habert, A. C. -Mater. Res., 5, p.37 (2002).

17. Ginn, B. T. \& Steinbock, O. - Langmuir, 19, p.8117 (2003). http://dx.doi.org/10.1021/la034138h

18. Svirachev, D. M. \& Tabaliov, N. A. - Bulg. J. Phys., 32, p.26 (2005)

19. Fleming, R. R.; Pardini, L. C.; Brito Junior, C. A. R.; Oliveira Junior, M. S.; Alves, N. P. \& Massi, M. - Polym. Bull., 66, p.282 (2010)

20. Owens, D. K. \& Went, R. C. - J. Appl. Polym. Sci., 13, p.1741 (1969). http://dx.doi.org/10.1002/app.1969.070130815

21. Sánchez, M. D.; Pastor-Blas, M. M. \& Martínez, J. M. M. - Int. J. Adhes. Adhes., 23, p.49-57 (2003).

Enviado: 02/10/12

Reenviado: 03/10/13 Aceito: 03/12/13 